\title{
DISTRIBUTION AND SEVERITY OF HYPOXIC-ISCHEMIC LESIONS ON BRAIN MRI FOLLOWING THERAPEUTIC COOLING: SELECTIVE HEAD VERSUS WHOLE BODY COOLING
}

\author{
S. Sarkar ${ }^{1}$, J.R. Bapuraj ${ }^{2}$, S.M. Donn ${ }^{1}$, I. Bhagat ${ }^{3}$, J.D. Barks ${ }^{1}$ \\ ${ }^{I}$ Department of Pediatrics, Division of Neonatal-Perinatal Medicine, ${ }^{2}$ Department of Radiology, University \\ of Michigan Health System, ${ }^{3}$ Department of Pediatrics, St. Joseph Mercy Hospital, Ann Arbor, MI, USA
}

Because whole body cooling (WBC) cools different regions of the brain uniformly, whereas selective head cooling (SHC) cools the superficial brain (cortex) more than the deeper brain (basal ganglia $\&$ thalamus, or BGT), we hypothesized that severity of hypoxic-ischemic (HI) lesions following cooling would differ between cooling modalities.

Objective: To compare the distribution and the severity of HI lesions on post-cooling brain MRI between infants treated with SHC or WBC.

Design/methods: 71 infants consecutively cooled by SHC $(n=34)$ or WBC $(n=37)$ underwent brain MRI at age 7-10 days. Images were evaluated by a masked neuroradiologist. Severity of involvement was assessed by a basal ganglia/watershed (BG/W) scoring system (as proposed by Barkovich and colleagues): 0, normal or no injury; 1, abnormal signal in BGT; 2, abnormal signal in cortex; 3, abnormal signal in cortex not extending beyond the watershed and BGT; 4, abnormal signal in cortex extending beyond the watershed areas and BGT.

Results: The 2 Groups had similar Apgar scores, initial blood pH, base deficit, early neurologic examination, and frequency of intrapartum sentinel event. Abnormal MRI was more frequent in the SHC group (SHC 25 of $34,74 \%$ versus WBC 18 of $37,49 \%$; $\mathrm{p}=0.05$, OR $2.9,95 \%$ CI 1.1-7.9). The SHC group also had more severe residual HI lesions (median BG/W Score: SHC 4 versus WBC 2, $\mathrm{p}=0.008$ ).

Conclusions: HI lesions on post-cooling brain MRI were more frequent and severe with SHC compared to WBC. This may reflect a selection bias with more severe cases receiving SHC. 